\title{
Dipping Pattern of Nocturnal Blood Pressure in Hypertensive Patients Treated with Azilsartan
}

\author{
P. L. N. Kapardhi, Viraj R. Suvarna', Rahul Chavda², Reshma Susan Reji ${ }^{3}$, Manoj A. Suva ${ }^{4}$ \\ ORCID: \\ P. L. N. Kapardhi: https://orcid.org/0000-0003-4158-7023 \\ Viraj R. Suvarna: https://orcid.org/0000-0002-2748-7723 \\ Rahul Chavda: https://orcid.org/0000-0002-1346-0634 \\ Reshma Susan Reji: https://orcid.org/0000-0001-5850-7080 \\ Manoj A. Suva: https://orcid.org/0000-0003-0484-7375
}

Department of Cardiology, Director Cath Lab, Century Hospitals, Hyderabad, Telangana, 'Department of Medical, Eris Lifesciences Ltd., ${ }^{2}$ Marketing Executive, Eris Lifesciences Ltd., ${ }^{3}$ Medical Advisor, Eris Lifesciences Ltd., ${ }^{4}$ Medical Services, Eris Lifesciences Ltd., Ahmedabad, Gujarat, India

\section{Abstract}

Aim: On average, blood pressure (BP) is found to be low during night than during day by approximately $10 \%-20 \%$. In addition, BP decreases by $>20 \%$ in some hypertensives or lowered by $<10 \%$ or few patients may experience rise in BP during night compared to daytime BP. This study was performed to determine the dipping pattern of BP variability of the patients receiving azilsartan with the help of ambulatory BP monitoring (ABPM) system. Settings: This was a prospective, observational, open-label, single-center study. Materials and Methods: A total of 158 hypertensive patients (systolic BP [SBP] $>140 \mathrm{mmHg}$; diastolic BP [DBP] $>90 \mathrm{mmHg}$ ) were enrolled to performed ABPM and clinic BP monitoring with ongoing treatment with azilsartan. Statistical Analysis: Data are expressed as mean \pm standard deviation. Statistical comparisons to test differences between two independent groups were by Student's $t$-test or Mann-Whitney's U-test as appropriate. Results: All patients monitored for 24-h BP measurement and reported to had SBP $133.76 \pm 15.97 \mathrm{mmHg}$ and DBP $76.16 \pm 10.86 \mathrm{mmHg}$. Pulse rate was found to be $76.18 \pm 11.82 \mathrm{bpm}$. BP variability was found to be high in $23.41 \%$ patients. Overall, study showed $34.17 \%$ dipper, $3.16 \%$ extreme dipper, $51.26 \%$ nondippers and $11.39 \%$ patients with reverse dipping pattern. In the present study, the dippers are classified to have $18.9 \%$ reduction in SBP, whereas $12.5 \%$ reduction in DBP. In the dippers group, only 4 patients had normal BP variation (2.53\%), whereas high BP variation was found in 50 patients of the group (31.64\%). Conclusions: ABPM had revealed higher SBP, BP variability (in almost one-fourth patients), dipping pattern and slightly higher pulse rates in hypertensive patients receiving azilsartan treatment. Around one-third of hypertensive patients were found to be dippers and more than half patients were non-dippers. Azilsartan has potent antihypertensive effect over $24 \mathrm{~h}$ and can be preferred in high-risk hypertensive patients.

Keywords: Ambulatory blood pressure monitoring, azilsartan, blood pressure variability, dipping, nondipping, reverse dipping

\section{INTRODUCTION}

Hypertension treatment has evolved over a period of time with a gradual shift from a step care regimen based on BP alone, to total risk-based assessment and management. Blood pressure (BP) is found to be low during night period (sleep) than during day period (waking time) by approximately $10 \%-20 \%$. In addition, BP decreases by $>20 \%$ in some hypertensive patients or lowered by $<10 \%$ in others, and few patients may experience rise in BP

Received: 16-04-2019 Revised: 23-07-2019 Accepted: 20-10-2019

Published Online: 11-06-2020

\begin{tabular}{|l|l|}
\hline \multicolumn{3}{c|}{ Access this article online } \\
\hline Quick Response Code: & Website: \\
& \\
http:/www.ijcva.com
\end{tabular}

during night compared to daytime BP. Recent guidelines on the treatment of hypertension have highlighted the importance of assessing the associated risk factors and the presence and extent of target organ damage along with BP measurements to decide the initiation of treatment and drug selection. ${ }^{[1]}$ As

Address for correspondence: Dr. P. L. N. Kapardhi, Department of Cardiology, Director Cath Lab, Century Hospitals, Road No.12, Banjara Hills, Hyderabad - 500 044, Telangana, India. E-mail:dr.kaparthi@gmail.com

This is an open access journal, and articles are distributed under the terms of the Creative Commons Attribution-NonCommercial-ShareAlike 4.0 License, which allows others to remix, tweak, and build upon the work non-commercially, as long as appropriate credit is given and the new creations are licensed under the identical terms.

For reprints contact:WKHLRPMedknow_reprints@wolterskluwer.com

How to cite this article: Kapardhi PL, Suvarna VR, Chavda R, Reji RS, Suva MA. Dipping pattern of nocturnal blood pressure in hypertensive patients treated with azilsartan. Int J Cardiovasc Acad 2020;6:41-5. 
compared to conventional clinical BP monitoring, ambulatory BP monitoring (ABPM) more accurately predict cardiovascular morbidity and mortality. ${ }^{[2]}$ Over the last decade, ABPM devices are increasingly used in the diagnosis of hypertension to exclude white coat hypertension, which affects about $15 \%-30 \%$ of the population and also useful in the diagnosis of patients with borderline hypertension, in looking at the efficacy of antihypertensive treatment and identifying nocturnal hypertension. ${ }^{[3]}$ Antihypertensive treatment leads to significant reduction in relative risk of cardiovascular events, although the number of patients needed to be treated (NNT) to prevent one cardiovascular event is substantially high, especially when the disease is mild. ${ }^{[4]}$ In a meta-analysis of 147 trials, the relative risk reduction of coronary artery disease was $21 \%$ over a mean of 4.6 years, for a BP reduction of $10 / 5 \mathrm{mmHg}$ (systolic/diastolic), among patients a pretreatment systolic $\mathrm{BP}$ (SBP) of 132-186 mmHg and without any cardiovascular disease (CVD). However, the NNT to prevent one event works out to around $140 .{ }^{[1]}$ This number will be larger if stage one hypertensive is considered. The goal of assessment and risk stratification is to identify high-risk hypertensive patients who would derive the maximum benefit from treatment compared to low-risk hypertensive whose benefit from antihypertensive treatment may not be favorable in terms of risk-benefit ratio, considering the long-term treatment side effects. ${ }^{[5,6]}$ While the presence of target organ damage is a definite indication for initiating treatment, physicians have been looking for simpler clinical tools to identify high-risk individuals. BP that does not dip down to the normal physiological range during the night has been identified as a reliable indicator of increased cardiovascular complications in the future and is emerging as an independent and powerful marker for high-risk hypertensive patients. ${ }^{[7]}$ Azilsartan is a novel angiotensin II receptor blocker (ARB) with potent BP-lowering effects. ${ }^{[8]}$

\section{The concept of dipper and nondipper}

In healthy individuals, BP follows a circadian pattern. BP starts declining from late evening onward, reaches a nadir around midnight, and shoots up just after awakening in the morning. Dips in BP have been described in three windows of sleep: BP starts declining in the visceral window, reaches the plateau level in the basal window, and rises in the preawakening window. ${ }^{[9]}$ This phenomenon of BP dipping has been documented by 24-h ABPM devices. This natural circadian variation may be altered by certain metabolic and cardiovascular changes. ${ }^{[10,11]}$ The present study was conducted with an objective to determine the dipping pattern BP variability of the hypertensive patients receiving azilsartan, with the help of ABPM system.

\section{Materials and Methods}

\section{Subjects}

The study included 158 hypertensive patients (age 35-60 years) without a history of major CVDs at baseline such as myocardial infarction, stroke, or congestive heart failure were receiving treatment with single-dose azilsartan (20 or $40 \mathrm{mg}$ depending on the patient current status of hypertension) for 6 months. Out of all patients, 62 females (39.24\%) and 96 males $(60.76 \%)$ were included and 22 patients $(13.92 \%)$ had diabetes. As it was prospective, open-label, observational study, no randomization or blinding or sample size calculation was done and patient selected without any bias. Patients with severe coexisting diseases, debilitating illness, dementia, and impairment of renal function were excluded from the study. The protocol conformed to the clinical guidelines of the local institutions and written informed consent was obtained from each patient. Patients were selected from the electrical chart. All patients were persistently hypertensive (more than $140 \mathrm{mmHg}$ SBP or $90 \mathrm{mmHg}$ diastolic BPs [DBP]) despite treatment with antihypertensive drug azilsartan.

\section{Home blood pressure measurements}

Initially, patients were informed how to measure their self BP and then instructed to record their BPs at least twice a week at home in the sitting position, once in the morning before breakfast within 30 min of awakening and again in the evening just before dinner using semi-automatic home BP measurement device that operates on the cuff oscillometric principle and generates a digital display of SBP and DBP and pulse rate. The accuracy of these self-measured BP monitoring readings was checked by well-trained clinic staff. A standard arm cuff was used to obtain the clinic and home BP monitoring readings because the circumference of patients' arm was $<14 \mathrm{~cm}$. These $\mathrm{BP}$ values were used as a reference for optimization of the dose of azilsartan. ${ }^{[12]}$

\section{Blood pressure monitoring with ambulatory blood pressure monitoring-05}

ABPM was performed using ABPM-05 device (Meditech Ltd., Hungary) which tracks BP and pulse rate, and it provides information on BP variability, overnight dipping, and morning surge for managing and controlling hypertension. ABPM-05 is a silent, compact, and very reliable ABPM. A large LCD screen ensures easy readability, and its lightweight design provides for maximum patient comfort. Using 2 AA rechargeable batteries ABPM-05 can make measurements up to a period of $48 \mathrm{~h}$. The device can store more than 600 readings in its solid-state memory for an unlimited length of time. Extra measurements can be triggered, and events can be marked manually. The device can be adjusted to the patient's lifestyle with a push of the day/night button. The study participants were instructed to hold the hand at heart level during the BP measurements. Central BP was assessed by applying the n-point moving average method, a mathematical low-pass filter, to the radial pulse waves. This method was validated against invasive measurements as well as against validated noninvasive methods for taking central pressure measurements. Nighttime was defined as from 9:00 PM to 6:00 AM. ${ }^{[13]}$

\section{Statistics}

Data are expressed as mean \pm standard deviation. Statistical comparisons to test differences between two independent 
groups were by Student's $t$-test or Mann-Whitney's U-test, as appropriate.

\section{Results and Discussion}

\section{Absolute rate systolic and diastolic pressure}

Defining normal ABPM BP values is difficult, and normal values are different in adults, children, and pregnant women. However, according to the most widely accepted criteria, normal daytime values are below $135 / 85 \mathrm{mmHg}$, while normal nighttime values are $<120 / 70 \mathrm{mmHg}$ and values are below $130 / 80 \mathrm{mmHg}$ for the whole $24-\mathrm{h}$ period in adults. A total of 158 patients were monitored for their 24-h SBP and DBP was calculated. 24-h mean variable of ABPM on azilsartan-treated patients is mentioned in Table 1. The number of patients with dipping status is shown in Figure 1.

The difference of values of mean systolic blood pressure and diastolic blood pressure between active and passive time

All the data of 158 patients were observed and the difference between the mean value of BP variable in the day (active time) and night time (passive time). There was a markable reduction

\begin{tabular}{lc}
\hline \multicolumn{2}{l}{ Table 1: 24-h mean variable of ambulatory blood } \\
pressure monitoring on azilsartan-treated patients \\
\hline Parameters & $\begin{array}{c}\text { Value of } 24 \mathbf{~ h} \\
\text { (mean } \mathbf{S D} \text { ) }\end{array}$ \\
\hline SBP & $133.76 \pm 15.97$ \\
DBP & $76.16 \pm 10.86$ \\
Pulse & $76.18 \pm 11.82$ \\
BP variability, number of & \\
patients $(n=158)$ & \\
High & 37 \\
Normal & 121 \\
Dipping status, number of & \\
patients $(n=158)$ & 54 \\
Dipper & 5 \\
Extreme dipper & 81 \\
Nondipper & 18 \\
Reverse dipping & \\
\hline$P<0.05$, not significant. SD: Standard deviation, BP: Blood pressure, \\
SBP: Systolic BP, DBP: Diastolic BP
\end{tabular}

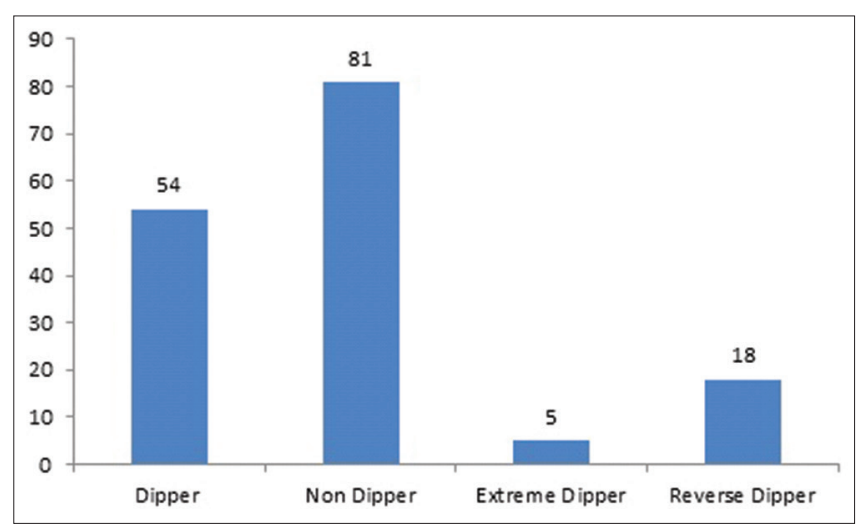

Figure 1: Patients with dipping status in the SBP and DBP reported in the dipping pattern, and there is a significant difference observed in the mean value of the same. Whereas, 18 patients reported to have higher mean passive time (night) BP with value 143/75.22 $\mathrm{mmHg}$ when compared to with that of the mean value of day (active) BP with value $136.56 / 73.11 \mathrm{mmHg}$. The number of patients with dipping status and BP variability is shown in Figure 2.

\section{Hypertensive subjects with dipping pattern (dippers)}

A total of 54 patients were reported to have the dipping pattern of BP of about $10 \%-20 \%$ decrease from the daytime BP values. In the present study, the dippers are classified to have $18.9 \%$ reduction in SBP and $12.5 \%$ reduction in DBP. In the dippers group, only four patients had normal BP variation, whereas high $\mathrm{BP}$ variation was found in 50 patients of the group. Mean BP variability on 24-h BP observation of dippers is shown in Table 2.

Hypertensive subjects without dipping pattern (nondippers) Total 81 patients were reported to have the dipping pattern of $<10 \%$ decrease in BP from daytime values. In the present study, the dippers are classified to have $7.1 \%$ reduction in SBP, whereas $6.7 \%$ reduction in DBP. In the dippers group, only four patients $(2.53 \%)$ had normal BP variation, whereas high BP variation was found in 50 patients (29.64\%) of the group. Dippers fail to follow the circadian rhythm which accounts to fall of BP by $\leq 10 \%$ and have the higher risk of cardiac-related damage. Mean BP variability on 24-h BP observation of nondippers is shown in Table 3.

\section{Hypertensive subjects with dipping pattern (extreme dippers)}

On the basis of the percentage of dipping status, patients were classified into different categories. In the present study, only five patients $(3.17 \%)$ were found to have higher BP dipping when compared to daytime BP. The mean SBP and DBP of the passive time were found to be reduced by $31.60 \%$ and $18.60 \%$, respectively. All of the patients had high BP variability and $60 \%$ patients had high morning surge.

\section{Hypertensive subjects with dipping pattern (reverse dippers)}

Unlike another dipping pattern, the patients of reverse dipping group had higher nighttime BP when compared

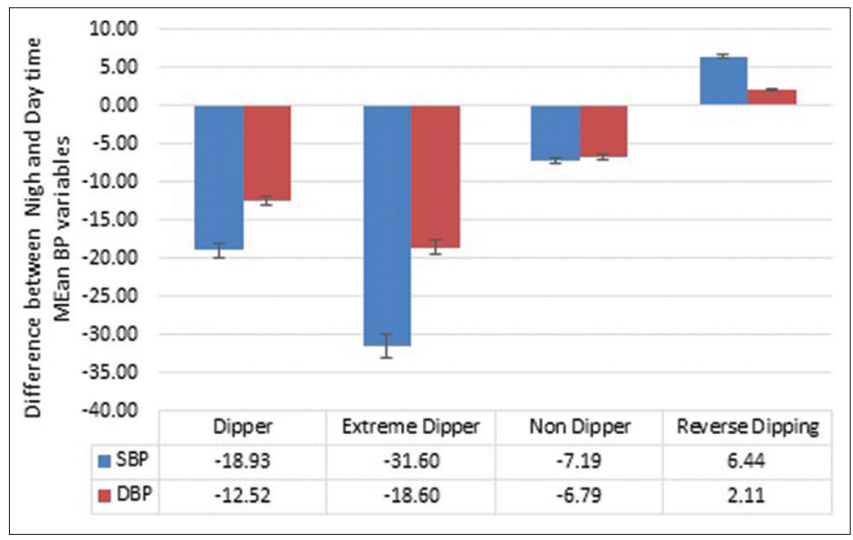

Figure 2: Patients with dipping status and blood pressure variability 
to that of active time. This may prognosis into a higher risk of organ failure and stroke. In the present study, 18 patients $(11.39 \%)$ were found to have reversed dipping pattern. Data are represented as mean BP variability on 24-h BP observation of reverse dippers in Table 4.

\section{Discussion}

Hypertensive patients were undergone 24-h ABPM, and differences between the mean value of BP variable in the day (Active time) and night (Passive time) were reported. Azilsartan at a dose of 20-40 $\mathrm{mg}$ has shown significant reduction in the SBP and $\mathrm{DB} P$ value in hypertensive patients

\begin{tabular}{lc}
\hline Table 2: Mean blood pressure variability on $\mathbf{2 4} \mathbf{~}$ \\
\hline Variable & Value (mean $\mathbf{S D}$ ) \\
\hline 24 mean SBP & $130.89 \pm 12.83$ \\
24 mean DBP & $75.43 \pm 10.42$ \\
Mean pulse & $77.89 \pm 12.54$ \\
BP variation, number of patients & \\
High & 50 \\
Normal & 4 \\
Morning surge, number of patients & \\
High & 14 \\
Normal & 40 \\
\hline
\end{tabular}

BP observation of dippers. SD: Standard deviation, BP: Blood pressure, SBP: Systolic BP, DBP: Diastolic BP

\begin{tabular}{lc}
\hline \multicolumn{2}{l}{ Table 3: Mean blood pressure variability on $\mathbf{2 4} \mathbf{h}$} \\
\hline Variable & Value (mean \pm SD) \\
\hline 24 mean SBP & $134.68 \pm 16.79$ \\
24 mean DBP & $77.57 \pm 10.27$ \\
Mean pulse & $75.91 \pm 11.15$ \\
BP variation, number of patients & \\
High & 54 \\
Normal & 27 \\
Morning surge, number of patients & \\
High & 9 \\
Normal & 72 \\
\hline
\end{tabular}

BP observation of nondippers. SD: Standard deviation, BP: Blood pressure, SBP: Systolic BP, DBP: Diastolic BP

\begin{tabular}{lc}
\hline Table 4: Mean blood pressure variability on $\mathbf{2 4} \mathbf{h}$ \\
\hline Variable & Value (mean $\mathbf{S D}$ ) \\
\hline 24 mean SBP & $138.83 \pm 17.76$ \\
24 mean DBP & $73.94 \pm 13.51$ \\
Mean pulse & $74.61 \pm 11.96$ \\
BP variation, number of patients & \\
High & 12 \\
Normal & 6 \\
Morning surge, number of patients & \\
High & 2 \\
$\quad$ Normal & 16 \\
\hline
\end{tabular}

BP observation of reverse dippers. SD: Standard deviation, BP: Blood pressure, SBP: Systolic BP, DBP: Diastolic BP and also reduced the dipping pattern. In accordance with a study conducted by Alfonso Mention Perez and Cao (2017) demonstrated that azilsartan once daily at dose up to $40 \mathrm{mg}$ (with exception of $2.5 \mathrm{mg}$ for clinic DBP) lead to significant reduction in SBP and DBP (based on both clinic trough and 24-h ABPM measurements) relative to placebo in patients with mild to moderate uncomplicated essential hypertension. Reductions in SBP and DBP with azilsartan 20 and $40 \mathrm{mg}$ were statistically superior to those observed with olmesartan $20 \mathrm{mg} \cdot{ }^{[14]}$ Azilsartan medoxomil is effective and safe ARB with a unique pharmacologic profile versus other antihypertensive agents including slowed angiotensin II type 1 receptor dissociation rates and improved receptor specificity. Studies have shown that azilsartan medoxomil $80 \mathrm{mg}$ once daily reduced BP by a greater extent than olmesartan and valsartan with similar safety and tolerability profile. ${ }^{[8]}$ This ABPM study revealed that hypertensive patients had higher SBP, BP variability (in almost one-fourth patients), dipping pattern, and slightly higher pulse rates. A study by Duggal et al. reported normal dipping pattern in $45 \%$ patients, nondipping in $46 \%$ patients, extreme dipping in $6 \%$ patients, and reverse dipping in 3\% of patients with diabetes and hypertension. ${ }^{[2]}$ Similar to this study findings, the present study also revealed the normal dipping pattern in $34.17 \%$ patients, nondipping in $51.26 \%$ patients, extreme dipping in $3.16 \%$ patients, and reverse dipping in $11.19 \%$ of patients in hypertensive patients. Around one-third of the hypertensive patients (34.17\% patients) were found to be dippers and more than half patients were nondippers. Azilsartan is a novel ARB which has a potent antihypertensive effect over $24 \mathrm{~h}$ can be preferred as antihypertensive drug as either monotherapy or in combination with other drugs in high-risk hypertensive effect. ${ }^{[15]}$

\section{Conclusions}

ABPM had revealed higher SBP, BP variability (in almost one-fourth patients), dipping pattern and slightly higher pulse rates in hypertensive patients receiving azilsartan treatment. Around one-third of hypertensive patients were found to be dippers and more than half patients were non-dippers. Azilsartan has potent antihypertensive effect over $24 \mathrm{~h}$ and can be preferred in high-risk hypertensive patients.

\section{Acknowledgment}

We are thankful to all the patients for providing their participation in the observational study and providing approval for sharing the results.

\section{Financial support and sponsorship Nil.}

\section{Conflicts of interest}

There are no conflicts of interest.

\section{RefERENCES}

1. Mahabala C, Kamath P, Bhaskaran U, Pai ND, Pai AU. Antihypertensive therapy: Nocturnal dippers and nondippers. Do we treat them differently? 
Vasc Health Risk Manag 2013;9:125-33.

2. Duggal A, Bal BS, Singh N. Study of dipping and non-dipping patterns in patients of type 2 diabetes mellitus with hypertension and its association with microalbuminuria. Ann Int Med Dent Res 2017;2:20-4.

3. Mohamed AL, Katiman E, Hassan JA. Ambulatory blood pressure monitoring profile as a useful prognostic tool in patients with primary hypertension. Malays J Med Sci 2003;10:76-83.

4. Wallis EJ, Ramsay LE, Jackson PR. Cardiovascular and coronary risk estimation in hypertension management. Heart 2002;88:306-12.

5. Tocci G, Valenti V, Sciarretta S, Volpe M. Multivariate risk assessment and risk score cards in hypertension. Vasc Health Risk Manag 2007;3:313-20.

6. Phillips RA, Xu J, Peterson LE, Arnold RM, Diamond JA, Schussheim AE, et al. Impact of cardiovascular risk on the relative benefit and harm of intensive treatment of Hypertension. J Am Coll Cardiol 2018;71:1601-10

7. Hamrahian SM. Pathophysiology of Hypertension. Available from: https://emedicine.medscape.com/article/1937383-overview. [Last updated on 2017 May 18; Last accessed on 2019 Aug 13].

8. Baker WL, White WB. Azilsartan medoxomil: A new angiotensin II receptor antagonist for treatment of hypertension. Ann Pharmacother 2011;45:1506-15.
9. O'Brien E. The circadian nuances of hypertension: A reappraisal of 24-h ambulatory blood pressure measurement in clinical practice. Ir J Med Sci 2007; 176:55-63.

10. Bloomfield D, Park A. Night time blood pressure dip. World J Cardiol 2015;7:373-6.

11. Grossman E. Ambulatory blood pressure monitoring in the diagnosis and management of hypertension. Diabetes Care 2013;36 Suppl 2:S307-11.

12. Suzuki H, Seki M. Effects of azilsartan in ambulatory patients on maintenance hemodialysis: Monitoring at night and at home. J Nephrol Ther 2014;S1 (007):1-4.

13. Jankowski P, Bednarek A, Olszanecka A, Windak A, Kawecka-Jaszcz K, Czarnecka D, et al. Twenty-four-hour profile of central blood pressure and central-to-peripheral systolic pressure amplification. Am J Hypertens 2013;26:27-33.

14. Perez A, Cao C. Azilsartan in patients with mild to moderate hypertension using clinic and ambulatory blood pressure measurements. J Clin Hypertens (Greenwich) 2017;19:82-9.

15. Kario K, Hoshide S. Rationale, study design, and implementation of the ACS1 study: Effect of azilsartan on circadian and sleep blood pressure as compared with amlodipine. Blood Press Monit 2014;19:123-8. 